\title{
Emergence of Approximate Translation Invariance in Finite Intervals as a Speed Selection Mechanism for Propagating Fronts
}

\author{
Stavros Theodorakis ${ }^{a}$ and Epameinondas Leontidis ${ }^{b}$ \\ a Physics Department, University of Cyprus, P.O. Box 2053\%, Nicosia 1678, Cyprus \\ ${ }^{b}$ Chemistry Department, University of Cyprus, P.O. Box 20537, Nicosia 1678, Cyprus
}

November 21, 2018

\begin{abstract}
We introduce a new velocity selection criterion for fronts propagating into unstable and metastable states. We restrict these fronts to large finite intervals in the comoving frame of reference and require that their centers be insensitive to the locations of the ends of the finite intervals, exhibiting thus effectively an approximate translation invariance. Only one monotonic front has this behavior and its velocity is the one that is physically selected. We present analytic results in the case of piecewise parabolic potentials, and numerical results in other cases.
\end{abstract}

PACS numbers: 82.40.Ck, 05.45.-a, 47.54.+r. 


\section{The selection principle}

In many systems rendered suddenly unstable, propagating fronts appear. The determination of the speed of front propagation into an unstable state has attracted attention recently, since it cannot be achieved by simply solving the differential equation in the comoving frame of reference on a one-dimensional infinite domain. Indeed, there are many such solutions on such a domain, even though the propagating front in practice always relaxes to a unique shape and velocity. Selection principles have thus been formulated to determine the observable front, without having to solve directly the partial differential equation of motion for a range of initial conditions. These principles have involved concepts of linear and nonlinear marginal stability, of structural stability and of causality[1], and all of them try to deal with the puzzle of the reduction of the multiple solutions to the single observed one. All these selection principles examine the wave from the viewpoint of the moving front, the corresponding wave equation being reduced then to an ordinary differential equation involving the speed $v$ of propagation. This speed has a unique value if the front is invading a metastable state, but not so for the case of invasion into an unstable state. The latter case has consequently attracted most of the attention.

The selection principles mentioned above have been based on the study of Fisher's dimensionless equation, $u_{t}=u_{x x}+f(u)$, on the interval $(-\infty, \infty)$ with $f(0)=f(1)=0$, the states $u=0$ and $u=1$ being unstable and stable fixed points respectively. Aronson and Weinberger[2] have shown that sufficiently localized initial conditions will evolve into an observable front propagating with speed $v^{*}$, provided $f>0$ on $(0,1)$. This selected speed $v^{*}$ is the lowest speed for which the above partial differential equation admits a monotonic front joining the stable

state $u=1$ to the unstable state $u=0$, and it satisfies $v^{*} \geq 2 \sqrt{f^{\prime}(0)}$. Any monotonic traveling wave solution $u(\xi)$ of Fisher's equation, with $\xi=x-v t$ being the coordinate in the comoving frame of reference, is a propagating front 
with speed $v>0$ and satisfies the "steady state" ordinary differential equation

$$
\frac{d^{2} u}{d \xi^{2}}+v \frac{d u}{d \xi}+f(u)=0
$$

with $u(-\infty)=1, u(\infty)=0$. Note that any speed $v>v^{*}$ will give a monotonic front, though not the observed one. The selection criteria seek to determine this lowest speed $v^{*}$, without solving the initial value problem, selecting thus one among the multiple possible fronts invading the unstable state. If the selected speed is determined by the linear order terms alone we have the pulled case $v^{*}=2 \sqrt{f^{\prime}(0)}$ (linear marginal stability). If linear analysis fails, we have the pushed case $v^{*}>2 \sqrt{f^{\prime}(0)}$ (nonlinear marginal stability). In the case of fronts invading a metastable state though, only one front is possible on the interval $(-\infty, \infty)$, and hence the selection is automatic[3].

We shall adopt here a unifying approach, showing that there exists a single selection principle at work for both the metastable and unstable cases, and for the regimes of linear and nonlinear marginal stability alike. This approach exploits the fact that the observed front is translationally invariant in the comoving frame of reference, even on a large finite interval, in the sense that its location is effectively independent of the ends of the interval. We solve then the steady state equation of motion on a large finite interval for an arbitrary given speed $v$, subject to the appropriate boundary conditions, obtaining a certain solution. This solution, however, will have approximate translational symmetry, thus becoming a physically observable front, only for a certain value of $v$. It is this value $v^{*}$ of $v$ that is experimentally observed. Thus the selected front is the one that is effectively translationally invariant on a large finite interval, in the comoving frame of reference.

Indeed, let us assume that $u(\xi)$ is the solution of Eq. (1) subject to the boundary conditions $u\left(L_{1}\right)=1$ and $u\left(L_{2}\right)=0$, with $L_{1} \ll L_{2}$. There is only one such solution for a given velocity $v$. We define the continuous potential $V(u)$, where $f(u)=-\partial V / \partial u$. We multiply Eq. (1) with $d u / d \xi$ and integrate from $L_{1}$ 
to $L_{2}$, obtaining then

$$
v=\frac{V(0)-V(1)-\frac{1}{2} w^{2}\left(L_{2}\right)+\frac{1}{2} w^{2}\left(L_{1}\right)}{\int_{L_{1}}^{L_{2}} w^{2}(\xi) d \xi},
$$

with $w(\xi)=d u / d \xi$. If $u(\xi)$ is going to be a physically observable front on this large, but finite, interval, it will have to be essentially translationally invariant. This means that $d u / d \xi$ will be effectively zero in the regions close to the boundaries, $u$ having reached a fixed point there. Consequently $w\left(L_{1}\right)$ and $w\left(L_{2}\right)$ will tend to zero, while $\int_{L_{1}}^{L_{2}} w^{2}(\xi) d \xi$ will be finite and practically independent of $L_{1}$ and $L_{2}$, as $L_{1} \rightarrow-\infty$ and $L_{2} \rightarrow \infty$. Hence the speed $v$ of Eq. (2) becomes independent of the endpoints of the interval, acquiring a unique value. In other words, only the front with that particular speed can be translationally invariant.

The requirement that the front be independent of the ends of the finite interval selects therefore the speed

$$
v^{*}=(V(0)-V(1)) / \int_{L_{1}}^{L_{2}} w^{2}(\xi) d \xi
$$

with $L_{1} \rightarrow-\infty$ and $L_{2} \rightarrow \infty$, as the speed of the physically observed front. Note that no distinction has been made here between metastable and unstable states. Indeed, given any particular speed $v$, we can find a front interpolating between the stable and the unstable or metastable state, as we shall demonstrate below, provided the solution is found on a finite interval. As the boundaries go to infinity, the value of the speed is restricted to $v^{*}$ and the front becomes the one corresponding to the speed of Eq. (3).

\section{Analytic examples}

We shall demonstrate the proposed selection principle through analytic and numerical work, both for the unstable and metastable cases. We shall adopt for our analytic work the continuous potential

$$
\begin{aligned}
V(u) & =\alpha\left(1-4 u^{2}\right) / 8 \quad \text { if } \quad 0 \leq u \leq 1 / 2, \\
& =\nu(1-2 u)(3-2 u) / 8 \quad \text { if } \quad u \geq 1 / 2,
\end{aligned}
$$


where $\nu>0$ and $\alpha=1$ or -1 , depending on whether the state $u=0$ is unstable or metastable, respectively. The corresponding $f(u)$,

$$
\begin{aligned}
f(u) & =\alpha u \quad \text { if } \quad 0 \leq u \leq 1 / 2 \\
& =\nu(1-u) \quad \text { if } \quad u \geq 1 / 2
\end{aligned}
$$

is piecewise linear and leads to exact analytic solutions. A similar piecewise linear model, but with $\xi$ in the interval $(-\infty, \infty)$, has been used for a discrete reaction-diffusion equation[4]. Piecewise parabolic potentials, always on the interval $(-\infty, \infty)$, have been used in nucleation and crystallization problems as well $[5]$.

Eq. (1) will be solved subject to the conditions $u\left(L_{1}\right)=1, u\left(L_{2}\right)=0$, where $L_{1} \ll 0 \ll L_{2}$. We shall assume that the midpoint of the front occurs at the point $\xi_{0}$, with $u\left(\xi_{0}\right)=1 / 2$, noting that both $u(\xi)$ and $d u / d \xi$ have to be continuous at that point. There are thus five boundary conditions that have to be satisfied, whereas the solution will involve five unknown parameters for any given value of $v$, namely $\xi_{0}$ and two constants for each linear piece of $f(u)$. We expect therefore a unique solution for each value of $v$.

Indeed, the exact solution of Eq. (1) for the $f$ of Eq. (5) is

$$
\begin{aligned}
u(\xi) & =1-\frac{1}{2} e^{v\left(\xi_{0}-\xi\right) / 2} \frac{\sinh \left[\sqrt{v^{2}+4 \nu}\left(\xi-L_{1}\right) / 2\right]}{\sinh \left[\sqrt{v^{2}+4 \nu}\left(\xi_{0}-L_{1}\right) / 2\right]} \quad \text { if } \quad L_{1} \leq \xi \leq \xi_{0} \\
& =\frac{1}{2} e^{v\left(\xi_{0}-\xi\right) / 2} \frac{\sinh \left[\sqrt{v^{2}-4 \alpha}\left(\xi-L_{2}\right) / 2\right]}{\sinh \left[\sqrt{v^{2}-4 \alpha}\left(\xi_{0}-L_{2}\right) / 2\right]} \quad \text { if } \quad \xi_{0} \leq \xi \leq L_{2}
\end{aligned}
$$

where $\xi_{0}$ satisfies

$2 v=\sqrt{v^{2}+4 \nu} \operatorname{coth}\left[\sqrt{v^{2}+4 \nu}\left(\xi_{0}-L_{1}\right) / 2\right]+\sqrt{v^{2}-4 \alpha} \operatorname{coth}\left[\sqrt{v^{2}-4 \alpha}\left(\xi_{0}-L_{2}\right) / 2\right]$.

These relations hold even if $v^{2} \leq 4 \alpha$. The solution of Eq. (7) gives $\xi_{0}$ as a function of the speed $v$.

(a) Unstable case : $\alpha=1$.

(i) We examine the case $v>2$ first. If we require $L_{1} \ll \xi_{0} \ll L_{2}$, then Eq. (7) reduces to $\sqrt{v^{2}-4}=\sqrt{v^{2}+4 \nu}-2 v$. This equation has a real solution, $v=v^{*}=$ 
$(\nu+1) / \sqrt{2 \nu-2}$, provided $\nu \geq 3$. In other words, for that particular value of $v$ the midpoint $\xi_{0}$ can be anywhere in the interval and cannot be determined, rendering thus the front effectively translationally invariant on the finite domain $\left[L_{1}, L_{2}\right]$. The value $v^{*}$ is therefore the selected speed if $\nu \geq 3$. In fact, the departures of $v$ from $v^{*}$ are miniscule and fall exponentially as the ends of the interval go to infinity.

Since $v^{*}>2$ when $\nu>3$, we have a pushed case. If however $\nu<3$, Eq. (7) yields

$$
\xi_{0} \approx L_{1}+2 \frac{\operatorname{coth}^{-1}\left[\left(2 v+\sqrt{v^{2}-4}\right) / \sqrt{v^{2}+4 \nu}\right]}{\sqrt{v^{2}+4 \nu}},
$$

and the front sticks to the left boundary.

(ii) Consider the case $v<2$ next. Eq. (6) shows then that on the interval $\left[\xi_{0}, L_{2}\right]$ the solution $u(\xi)$ is proportional to $\sin \left[\sqrt{4-v^{2}}\left(\xi-L_{2}\right) / 2\right]$ and has multiple roots. Our front though has to be monotonic, with no overshooting occurring. Thus $u(\xi)$ must become zero only on the boundary $L_{2}$. Therefore the point $r=L_{2}$ $-2 \pi / \sqrt{4-v^{2}}$, where the above sine becomes zero, must lie outside the interval $\left[\xi_{0}, L_{2}\right]$, and hence $\xi_{0}>r$. In that case Eq. (7) yields

$\xi_{0}=L_{2}-\frac{\pi}{\sqrt{4-v^{2}}}+\frac{2}{\sqrt{4-v^{2}}} \tan ^{-1}\left[\frac{-2 v+\sqrt{v^{2}+4 \nu} \operatorname{coth}\left[\sqrt{v^{2}+4 \nu}\left(\xi_{0}-L_{1}\right) / 2\right]}{\sqrt{4-v^{2}}}\right]$.

This exact equation holds for $v<2$ and all values of $\nu$, and determines the location $\xi_{0}$ of the midpoint of the monotonic front. Thus Eqs. (7) and (9) determine fully $\xi_{0}$ for a given $v$. In fact, if $\nu<3$ and $v$ is just below 2, Eq. (9) yields

$$
\xi_{0} \approx L_{2}-2 \pi / \sqrt{4-v^{2}}-(\sqrt{\nu+1}-2)^{-1}
$$

or equivalently

$$
v \approx \sqrt{4-4 \pi^{2} /\left[L_{2}-\xi_{0}+(2-\sqrt{\nu+1})^{-1}\right]^{2}} .
$$

Thus $v$ has a plateau as a function of $\xi_{0}$ at the value $v=2$, if $\nu<3$.

Fig. (1) shows $v$ as a function of the midpoint $\xi_{0}$ for the cases $\nu=9$ and $\nu=2$, for the potential of Eq. (4) with $\alpha=1$. For $\nu=9$, the selected speed is $v^{*}=2.5$ 
(pushed case). We see that for $v>2.5$ the front is located close to $L_{1}$, while it shifts abruptly to $L_{2}$ when the speed becomes less than $v^{*}$. When $v$ is equal to $v^{*}$, the graph has a plateau, indicating that $\xi_{0}$ is pretty much undetermined, the solution being thus effectively translationally invariant. For the case $\nu=2$, on the other hand, the front is located at $L_{1}$ when $v>2$, as indicated by Eq. (8), while for $v$ just below 2 the location of the front shifts abruptly to $L_{2}$, as indicated by Eq. (10). We see thus the appearance of a plateau at $v=2$, if $\nu=2$. For that value of $v$ the front's location is rather undetermined, indicating that the front has acquired effectively a translational invariance. The selected speed is thus $v^{*}=2$ (pulled case).

Our analytic example indicates then that for $\nu>3$ we have the pushed case, the selected speed being $(\nu+1) / \sqrt{2 \nu-2}$, while for $\nu \leq 3$ we have the pulled case, the selected speed being $v^{*}=2$. In both cases the selected speed corresponds to a plateau in the graph of $v$ versus $\xi_{0}$, due to the emergence of an approximate translational invariance of the front at that speed.

(b) Metastable case : $\alpha=-1$.

If $\nu>1$ the stable state is at $u=1$ and the metastable one at $u=0$. The boundary conditions are once more $u\left(L_{1}\right)=1, u\left(L_{2}\right)=0, u\left(\xi_{0}\right)=1 / 2$, along with continuity of $u$ and $d u / d \xi$ at $\xi_{0}$. For a given value of $v$ there are then five boundary conditions and five unknown parameters, two constants for each linear piece plus $\xi_{0}$. There is thus a single solution of Eq. (1) for any given value of $v$. This is the solution given by Eqs. (6) and (7), but with $\alpha=-1$. We can show that Eq. (7) reduces to $v=v^{*}=(\nu-1) / \sqrt{2 \nu+2}$ if $L_{1} \ll \xi_{0} \ll L_{2}$. In other words, for that particular value of $v$ the midpoint $\xi_{0}$ can be anywhere in the interval, leaving the location of the front undetermined. The front acquires thus an effective translational invariance at that speed. This translational invariance is recognised in Fig. (2a), where $\nu=7$, as a plateau in the graph of $v$ versus $\xi_{0}$ at the speed $v^{*}=1.5$.

Note that there is a solution to the metastable problem for any $v$ on a finite 
domain in the comoving frame of reference, but only for one $v$ on the infinite domain. Indeed, if the metastable problem is solved on $(-\infty, \infty)$, then $\xi_{0}$ cannot be determined due to the exact translational symmetry, leaving us thus with five boundary conditions but only four unknown parameters, two for each linear piece. Consequently $v$ will also have to be considered as a parameter to be determined, giving thus a solution only for a unique value of $v[3]$, which is precisely the one selected by our selection principle. On a semi-infinite domain, on the other hand, a continuum of values is possible for $v[6]$.

In the unstable case, exact translational symmetry on $(-\infty, \infty)$ reduces again the number of unknown parameters by one $\left(\xi_{0}\right)$, but the boundary condition at $u=0$ is trivially satisfied due to the existence of two decaying exponentials, and thus we are left with four boundary conditions and four unknown parameters, two for each linear piece, for a given value of $v$. Solutions are thus possible for a continuum of values of $v$.

\section{Numerical examples}

We can demonstrate our selection principle numerically as well, for the case

$$
f(u)=u(b+u)(1-u) / b
$$

where for $0<b<1$ the states $u=-b, u=0$ and $u=1$ are metastable, unstable and stable, respectively. In fact, it was this particular choice of $f(u)$ that was used when the concepts of linear and nonlinear marginal stability were first proposed[7]. That study found that for $1>b>1 / 2$ the selected speed for the front invading the unstable state is $v^{*}=2$, while for $0<b<1 / 2$ it is $v^{*}=(2 b+1) / \sqrt{2 b}$. For the front invading the metastable state the selected speed was found to be $(1-b) / \sqrt{2 b}$.

We have solved Eq. (1) numerically on a finite $\xi$ domain for the $f(u)$ of Eq. (11), with $b=1 / 8$, subject to the boundary conditions $u\left(L_{1}\right)=1, u\left(L_{2}\right)=-b$ (metastable case). We found that the plot of $v$ versus the characteristic point $\xi_{0}$ 
of the front, where $\xi_{0}$ is defined through the relation $u\left(\xi_{0}\right)=1 / 2$, has a plateau at $v=1.75$ (see Fig. 2b), indicating that at that speed the solution has become approximately translationally invariant on the finite $\xi$ domain. The speed on the plateau is precisely the one selected by the marginal stability criterion[7].

Furthermore, we have solved Eq. (1) numerically on a finite $\xi$ domain for the $f(u)$ of Eq. (11), with $b=1$ and $b=1 / 8$, subject to the boundary conditions $u\left(L_{1}\right)=1, u\left(L_{2}\right)=0$ (unstable case). We find again that the plot of $v$ versus $\xi_{0}$, where $u\left(\xi_{0}\right)=1 / 2$, has a plateau at $v=2$ and $v=2.5$, respectively (see Fig. 3 ), indicating that the solution acquires effectively translational invariance there. These values are once again the ones known to be selected[7].

\section{Concluding remarks}

We see then that requiring the solution to have approximate translational invariance on a finite interval in the comoving frame of reference results in the selection of a speed for the front in both the metastable and unstable cases. This speed is precisely the one given by marginal stability. A similar selection of a single velocity occurs when a cutoff is introduced, albeit on an infinite domain[8]. The importance of the translational invariance has also been noted in connection with the precursors of the propagating fronts [9]. Indeed, the selected solution is the only one with a legitimate translation mode in its stability spectrum. Thus one way of understanding the marginal stability of the selected solution is through

requiring that the stability operator of a physically realizable solution possess a translation zero mode.

We can adopt then a selection principle that reads "the selected front is the one that is approximately translationally invariant on a large finite interval, with respect to the comoving frame of reference". This principle is very easy to implement for both the metastable and unstable cases, especially numerically. Indeed, it suffices to solve the comoving frame equation on a large finite interval. For large speeds we expect the midpoint of the front to be close to the left boundary. As 
the speed $v$ is lowered, the midpoint suddenly moves to the right boundary. The speed $v^{*}$ at which this sudden move occurs is the speed selected by the physically observed front. 


\section{References}

[1] Wim van Saarloos, Phys. Rev. 39A, 6367 (1989); G.C. Paquette, L.-Y. Chen, N. Goldenfeld, Y. Oono, Phys. Rev. Lett. 72, 76 (1994); G.C. Paquette and Y. Oono, Phys. Rev. 49E, 2368 (1994); J.-M. Chomaz and A. Couairon, Phys. Rev. Lett. 84, 1910 (2000); X.Y. Wang, S. Fan and T. Kyu, Phys. Rev. 56E, R4931 (1997).

[2] D.G. Aronson and H.F. Weinberger, Adv. Math. 30, 33 (1978).

[3] M.C. Cross and P.C. Hohenberg, Rev. Mod. Phys. 65, 851 (1993).

[4] Gabor Fath, Physica 116D, 176 (1998).

[5] L. Granasy and D.W. Oxtoby, J. Chem. Phys. 112, 2399 (2000); L. Granasy and D.W. Oxtoby, J. Chem. Phys. 112, 2410 (2000); C.K. Bagdassarian and D.W. Oxtoby, J. Chem. Phys. 100, 2139 (1994); D.W. Oxtoby and P.R. Harrowell, J. Chem. Phys. 96, 3834 (1992); P.R. Harrowell and D.W. Oxtoby, J. Chem. Phys. 86, 2932 (1987); H. Lowen and D.W. Oxtoby, J. Chem. Phys. 93, 674 (1990).

[6] T. Meister and H. Muller-Krumbhaar, Phys. Rev. Lett. 51, 1780 (1983).

[7] E. Ben-Jacob, H. Brand, G. Dee, L. Kramer and J.S. Langer, Physica 14D, $348(1985)$.

[8] E. Brunet and B. Derrida, Phys. Rev. 56E, 2597 (1997).

[9] David A. Kessler, Zvi Ner, Leonard Sander, Phys. Rev. 58E, 107 (1998). 


\section{Figure Captions}

Figure 1: The speed $v$ as a function of the midpoint $\xi_{0}$ of the front invading the unstable state, for the $f(u)$ of Eq. (5), with $L_{1}=-15$ and $L_{2}=15$. The plateau is at $v=2.5$ for $\nu=9$, and at $v=2$ for $\nu=2$. All quantities are dimensionless.

Figure 2: (a) The speed $v$ as a function of the midpoint $\xi_{0}$ of the front invading the metastable state, for the $f(u)$ of Eq. (5), with $\nu=7, L_{1}=-15$ and $L_{2}=15$. The plateau is at $v=1.5$. (b) The speed $v$ as a function of the point $\xi_{0}$ of the front invading the metastable state, for the $f(u)$ of Eq. (11), with $b=1 / 8, L_{1}=-15$ and $L_{2}=15$. The plateau is at $v=1.75$.

Figure 3: The speed $v$ as a function of the midpoint $\xi_{0}$ of the front invading the unstable state, for the $f(u)$ of Eq. (11), with $L_{1}=-15$ and $L_{2}=15$. The plateau is at $v=2$ for $b=1$, and at $v=2.5$ for $b=1 / 8$. 


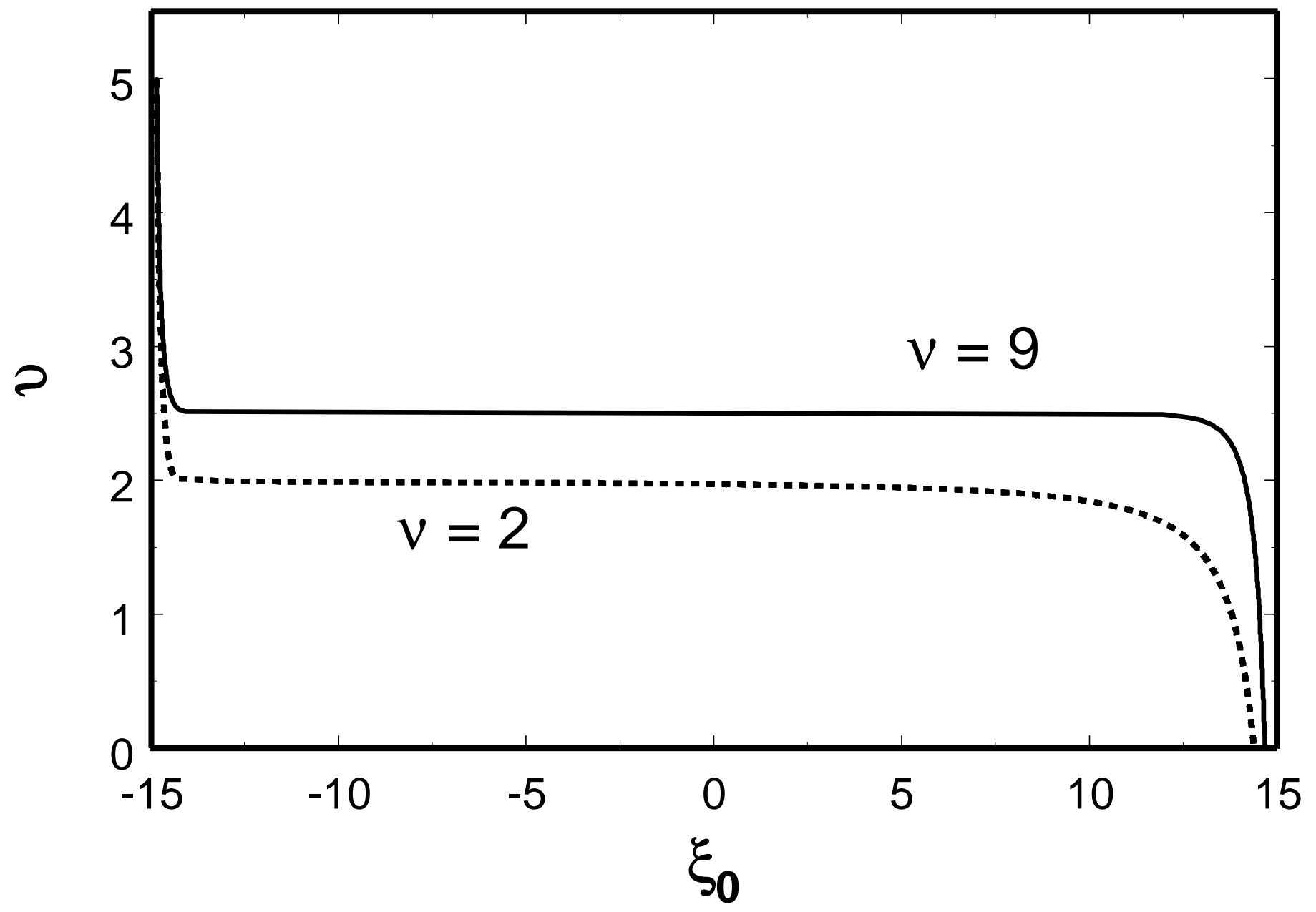




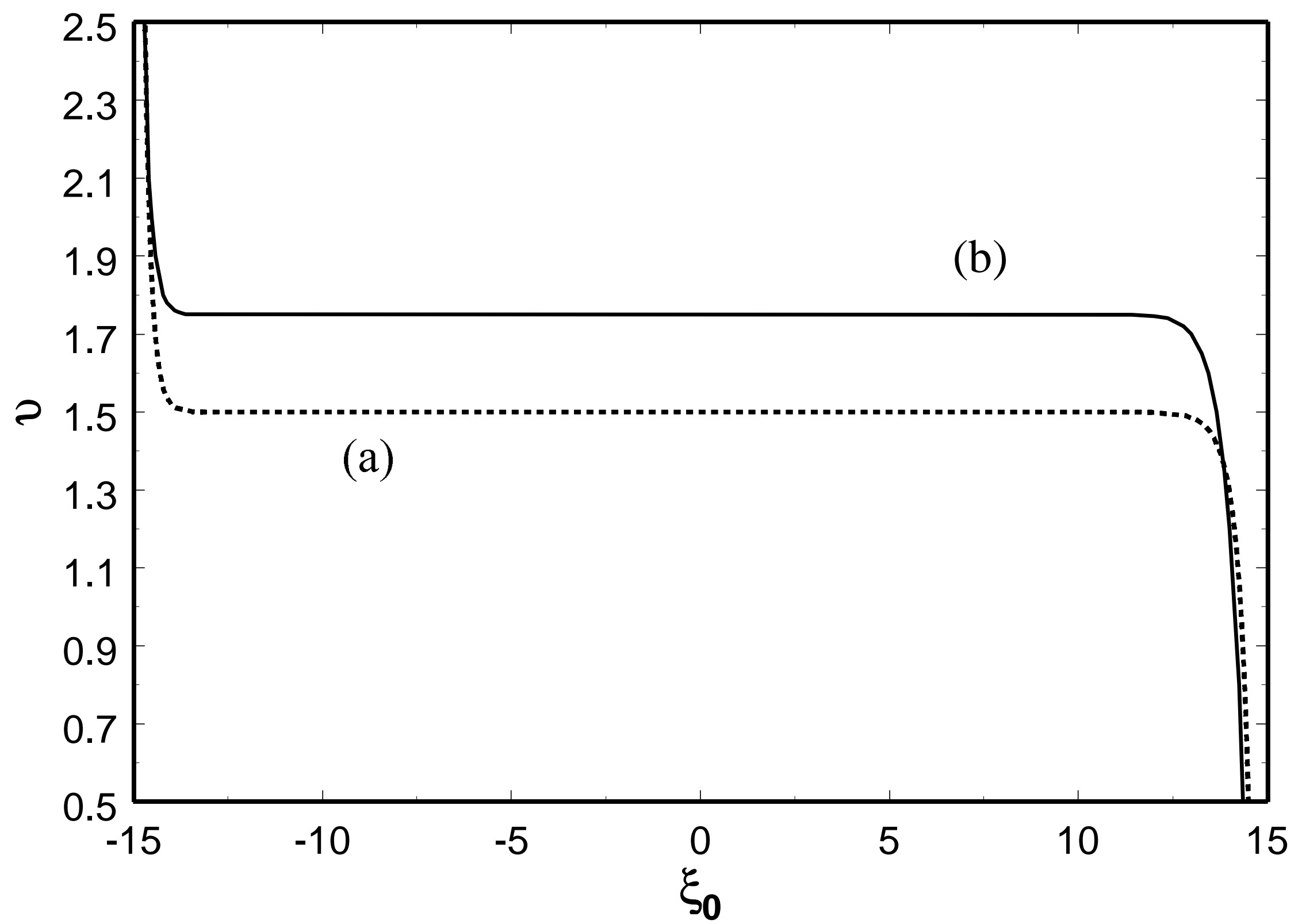




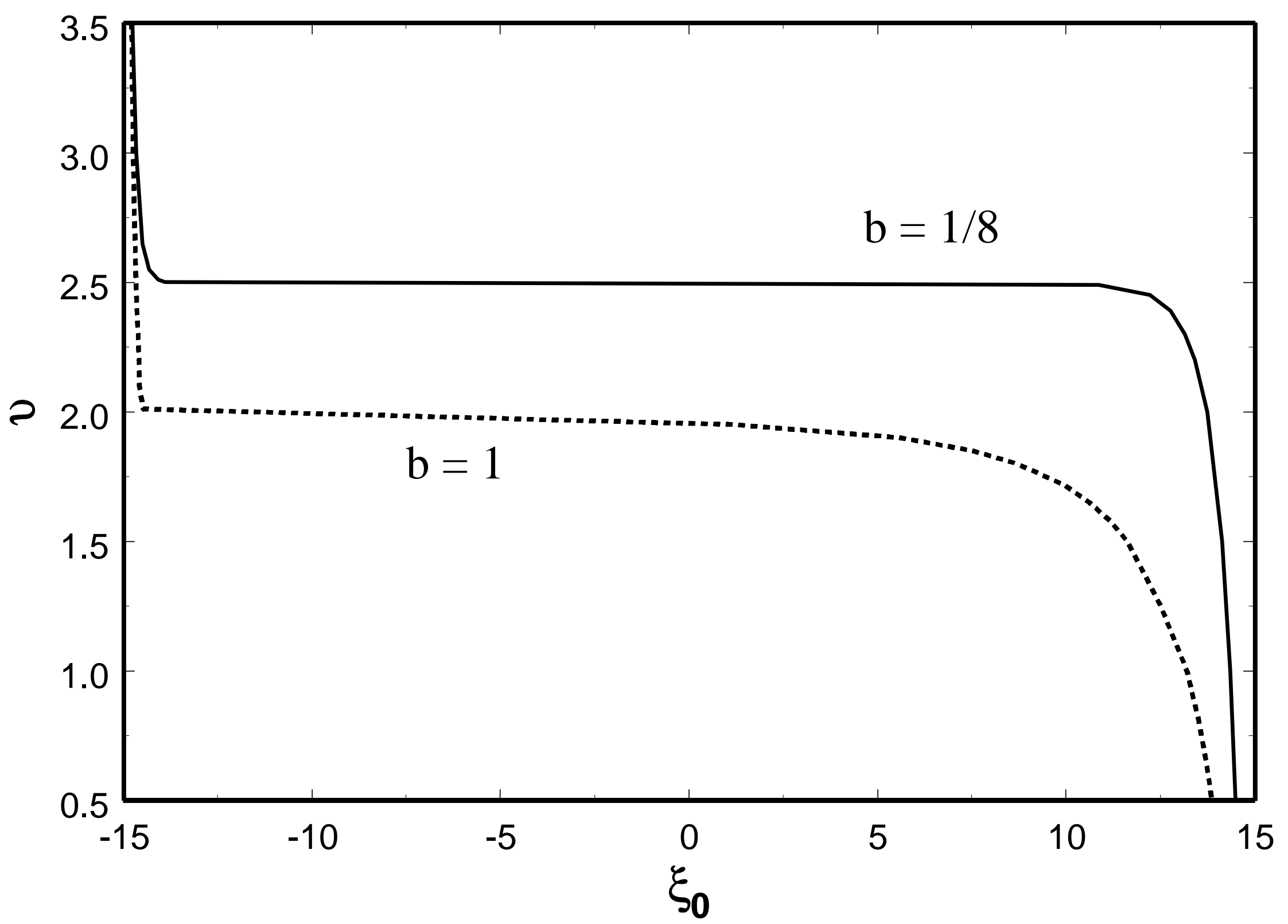

